\title{
MORFOLOGÍA CRANEOFACIAL EN PADRES DE NIÑOS CON LABIO Y PALADAR HENDIDO *
}

Olga Lucía Cañón J., ${ }^{2}$ Victoria Eugenia Gutiérrez $N$.

${ }^{1}$ Odontóloga, U. Santo Tomás, Especialista en Odontopediatría U. Javeriana,

Residente III año Especialización en Ortodoncia U. Santo Tomás, Docente U. Santo Tomás

${ }^{2}$ Odontóloga, U. Santo Tomás, Residente III año Especialización en Ortodoncia U. Santo Tomás

Autor responsable de correspondencia: Olga Lucía Cañón J.

correo electrónico: olluca17@yahoo.com

\section{RESUMEN}

objetivo: Identificar en los padres las medidas cefalométricas que pueden ser usadas como predictores de la posibilidad de tener hijos con labio y/o paladar hendido.

Materiales y Métodos: Mediante un estudio observacional descriptivo, se tomó por conveniencia una muestra de 83 padres y madres de hijos con hendiduras orofaciales que asistieron a la Fundación Operación Sonrisa en Bogotá y Bucaramanga, y 23 padres y madres de hijos sin hendiduras orofaciales que vivían en Bucaramanga. A cada uno se le tomó una radiografía lateral en la que se tuvieron en cuenta 12 medidas lineales y angulares para el análisis. Se evaluó el nivel de reproducibilidad interevaluador mediante el análisis del acuerdo de Bland y Altman. Para comparar las medidas cefalométricas entre los grupos de estudio se aplicó prueba $t$ de Student para comparar entre los dos grupos de estudio y ANOVA para comparar los promedios de medidas cefalométricas por tipo de hendidura, considerando un nivel de significancia de $\alpha=0.05$.

Resultados: Las medidas cefalométricas que se encontraron diferentes entre los dos grupos de estudio fueron: Co - A, ENA - ENP, Go-Me, S - N, Angulo SNB y todas estaban disminuidas de manera estadísticamente significativa $(\mathrm{P}<0.05)$ en el grupo de madres cuyos hijos registraban labio y paladar fisurado. No se observaron diferencias estadísticamente significativas en las medidas de los padres.

Conclusión: Se podría sugerir una mayor ingerencia genética materna en la morfología craneofacial. [Cañón oL, Gutiérrez VE. Morfología craneofacial en padres de niños con labio y paladar hendido. Ustasalud Odontología 2006; 5: 85 - 92]

Palabras clave: Niños con labio y paladar fisurado, Morfología craneofacial, Padres.

\section{CRANIOFACIAI, MORPHOLOGY OF THE PARENTS OF CHILDREN WITH LIP AND/OR CLEFT PALATE *}

\begin{abstract}
Purpose: To identify in the parents the cephalometric parameters that can be used to predict the chance of having children with lip and/or cleft palate.

Material and Methods: It was a descriptive observational study, with sample of 83 fathers and mothers of children with orofacial clefting that attended the Fundación Operación Sonrisa in Bogotá and Bucaramanga, and 23 fathers and mothers of children without orofacial clefting that lived in Bucaramanga. To each one a lateral x-ray was taken and 12 linear and anguiar measures were analysed. In order to compare the cephalometric measurements between the groups test $t$ of Student was applied. To compare between both ANOVA and training groups to compare the averages of cefalométricas measures by type of orofacial clefting, considering a level of significance of $\alpha=0.05$.

Results: The cephalometric measures that were different between the two groups were: Co - To, ENA - ENP, Go- Me, S - N, angle SNB and all were diminished of statistically significant difference $(\mathrm{P}<0.05)$ in the group of mothers whose children registered lip and cleft palate. There were no statistically significant differences in the measures of the fathers. Conclusion: A greater maternal genetic interference in the craniofacial morphology could be suggested.
\end{abstract}

Key words: Children with lip and/or cleft palate, orofacial clefting, Parents.

* Grupo de Investigación CYDUSTA. 


\section{INTRODUCCIÓN}

Las hendiduras orofaciales, específicamente el labio y/o paladar fisurado (LPH-PH), son las malformaciones más comunes del complejo craneofacial y el segundo defecto de nacimiento más frecuente después del síndrome de Down. ${ }^{1}$ De cada mil niños nacidos vivos en Colombia, entre 7 u 8 nacen con Labio y Paladar Hendido, sin que se pueda describir claramente la etiología de la malformación. ${ }^{2}$

Numerosos estudios han demostrado que las hendiduras orales tienen un componente poligénico y multifactorial, ${ }^{1-7}$ la evidencia indica que algunos de los genes asociados a hendiduras orofaciales son morfogenes craneofaciales; por lo tanto, se podría pensar que la detección de algunas variaciones en la morfología craneofacial de los padres de niños con fisuras orales ayudarían a predecir dichas fisuras.

Investigaciones previas como las desarrolladas por Fraser y Pashayan (1970) y en las que se estudió la morfología facial de padres de niños con hendiduras orales, se observó que éstos tenían una maxila poco desarrollada, diámetros bizigomáticos amplios y labio superior delgado, al compararlos con los controles. ${ }^{8}$ Coccaro y colaboradores, en 1972, usaron la cefalometría lateral para comparar los parámetros craneofaciales en padres con y sin LPH. Concluyeron que estos padres tenían medidas cefalométricas verticales y horizontales más cortas en cuanto a la parte superior de la cara, una longitud nasal más corta y la mandíbula tenia una tendencia prognatita reduciendo la convexidad del perfil. ${ }^{9}$

Posteriormente, los estudios de Kurisu y colaboradores (1974), Shibasaki y Ohtsuka (1978), Nakasima y Ichinose (1983), Prochazkova and Tolarova (1986) encontraron que no había relación en los rasgos que caracterizaban a los padres de LPH. 10 Sin embargo, en un estudio más reciente, Prochazkova y Vinsova (1995), examinaron 52 padres de niños con hendiduras y los compararon con un grupo control; de los padres de niños con LPH, 32 variables eran significativamente diferentes de los grupos controles y 25 variables en las madres eran también diferentes. ${ }^{11}$

El objetivo del presente estudio fue identificar las medidas cefalométricas que pueden ser usadas como predictores de la posibilidad de tener hijos con labio y/o paladar hendido.

\section{MATERIALES Y MÉTODOS}

Se realizó un estudio observacional descriptivo de corte transversal, donde se determinaron diferencias en la morfología craneofacial de los padres de niños con labio y/o paladar hendido vinculados con la Fundación Operación Sonrisa de Bogotá y Bucaramanga, y los padres de niños sin fisuras orales residentes en la ciudad de Bucaramanga.
La población se definió por conveniencia y estuvo conformada por dos grupos de estudio:

Grupo 1: 23 padres y/o madres que al momento de ser incluidos en el estudio, registraran no tener hijos con hendiduras orales, residentes en la ciudad de Bucaramanga, desde enero de 2003 a marzo de 2004.

Grupo 2: 83 padres y/o madres de individuos con labio y/o paladar hendido no sindrómico, que asistieron a consulta a las clínicas de la Fundación Operación Sonrisa en Bogotá y Bucaramanga, durante el mismo periodo del estudio.

Los criterios de inclusión considerados para cada uno de los grupos fueron:

Grupo 1: Padres sin enfermedades sindrómicas, ni enfermedades metabólicas que afecten de manera evidente su crecimiento, con hijos sin hendiduras orofaciales.

Grupo 2: Padres sin enfermedades sindrómicas, ni enfermedades metabólicas que afecten de manera evidente su crecimiento, con hijos que presenten labio y/o paladar hendido no sindrómicos.

Adicionalmente, se consideró para los dos grupos que los padres y madres en el momento del estudio fueran mayores de 18 años.

Se excluyeron del estudio los padres con algún síndrome y algún tipo de hendidura o cuyos hijos tengan LPH asociado a síndromes, condiciones que alteran las características cefalométricas y las madres con sospecha de embarazo o que se encontraban embarazadas al momento de estudio.

Las variables consideradas en el estudio se recopilaron en un instrumento diseñado para este fin y que incluía:

- Variables sociodemográficas: Género, estrato socioeconómico, familiares con LPH-PH, relación del familiar con LPH-PH (hermano, tío, abuelo, etc), tipo de hendidura (catalogada como: tipo1: LPH derecho, tipo 2: LPH izquierdo, tipo3: LPH Bilateral, tipo 4: Labio hendido derecho, tipo 5: Labio hendido izquierdo, tipo 6: labio hendido bilateral y tipo 7: Paladar hendido), contacto con tóxicos.

- Variables cefalométricas: Las medidas lineales y angulares usadas se definieron por ser las medidas que describen las regiones anatómicas de cara y cabeza haciendo énfasis en las áreas que podrían tener algún interés en el contexto del evento de estudio, son puntos cefalométricos fácilmente identificables y por ser las utilizadas en otros estudios. ${ }^{8,10,12,13,14}$

Las medidas cefalométricas del maxilar superior incluyeron: Longitud maxilar Co-A, Longitud maxilar ENA-ENP, altura superior N-ENA. 
Mandibulares: Longitud mandibular $\mathrm{Co}_{-} \mathrm{G}$, Iongitud mandibular Go-Me, altura de la rama Ar-Go, longitud base de cráneo S-N, altura inferior ENA-Me y que se recolectaron en escala de razón operacionalizada en milímetros.

Las medidas angulares incluidas fueron: ángulo goniaco Ar-GoGn, ángulo SNA, SNB, ANB que se recolectaron en grados.

Se realizó una prueba piloto en la que se verificaron los instrumentos, se estandarizaron los procesos de toma de las radiografias, se definieron tiempos de trabajo y se efectuaron los correctivos necesarios. Se calibró en procesos relacionados con los trazos cefalométricos y en la definición de técnicas y puntos de referencia con el propósito de homogenizar las mediciones entre los evaluadores.

Posterior a la prueba piloto, se ubicaron los padres de los pacientes con y sin labio y paladar hendido que consultaron a las clínicas odontológicas de la Universidad Santo Tomás y de la Fundación Operación Sonrisa, durante los años 2003 y 2004.

Se procedió a aplicar una encuesta para la selección de padres, explicándoles el proceso a seguir y quienes de manera voluntaria desearon participar en el estudio firmaron una carta de consentimiento informado y respondieron un cuestionario de datos generales, antes de proceder a la toma de las radiografias laterales.

Las radiografias laterales de cráneo para los pacientes tanto de Bogotá como en Bucaramanga fueron realizadas en centros radiológicos especializados por una persona especializada y siguiendo el mismo protocolo. En Bogotá el equipo empleado tenía las siguientes especificaciones: Panerex J Morita Corporation X 100EC-9405 Grid Ratio 5/1, Distancia Focal $130 \mathrm{~cm}$, Grid Lines 34 lines/cm, Interspacer aluminium. Las especificaciones del equipo usado en Bucaramanga fueron: Panoraura Ultra Pan/ Ceph X - Ray Apparatus. Modelo P.A 812/PA 812L. Output: 70-90 Kup 6 MA (Panoramic) y 10 MA (Céphalo). X- Ray tube SXR-15 (Sxt co, 1 rd) Tubo No yeso 546. Focus $10 \mathrm{~mm}$. Filtración $2,8 \mathrm{~mm}$ AL, Serial № LE -015. Fecha de fabricación Mayo de 2001. Certificación pomplies with DHHS radiation performance standards 21 CFR subchapter. The Yoshida Dental MFG. Co, ltd 1-3-6 Kotobashi Sumida, KV, Tokyo.

El proceso de toma de radiografias se estandarizó de la siguiente manera: Plano de Frankfort paralelo al piso, distancia del paciente al foco $152.4 c c$, paciente en oclusión habitual sin forzar el selle labial, el rayo central con dirección al conducto auditivo externo, perpendicular al plano de la película y al plano sagital medio.

Las medidas se trazaron en papel especial para cefalometrías con portalápiz de mina de $0,5 \mathrm{~mm}$ de grosor HB. Las radiografias fueron trazadas y cada una de las evaluadoras realizo una medición en dos ocasiones diferentes. Cuando las medidas arrojaron datos diferentes, se saco un promedio.
Para el plan de análisis, inicialmente, se describió la población de estudio según variables sociodemográficas, aplicando medidas de tendencia central y dispersión o tablas de frecuencia según la naturaleza y distribución de cada variable.

Se evaluó la reproducibilidad entre evaluadores, de las medidas cefalométricas aplicando la metodología de Bland y Altman.15 Posteriormente, se consideraron los promedios de las dos mediciones, para comparar las medidas cefalométricas entre los dos grupos de estudio, para lo cual se aplicó la prueba t de Student.16 Para comparar el promedio de cada una de las medidas cefalométricas lineares y angulares según tipo de hendidura se realizó análisis de varianza de una sola vía (ANOVA).

La base de datos se elaboro en Excel y se exporto a STATA 8.0 para su análisis final. Todo el análisis considero un nivel de significancia de $\mu .0 .05$.

Criterios éticos: Este estudio es catalogado según la Res. 008430/ 93 del Ministerio de Salud, como una investigación con riesgo mínimo. A cada padre se le entregó una carta de consentimiento informado para su firma. Los padres participaron y firmaron el consentimiento informado voluntariamente. Se les explicó de manera completa y detallada los objetivos y procedimientos a seguir en el presente estudio y prevaleció el criterio de respeto a su dignidad y la protección a sus derechos y su bienestar.

\section{RESULTADOS}

En total se evaluaron padres y/o madres de 76 niños y niñas, 15 niños del grupo 1 y 61 del grupo 2, la distribución por género mostró 8 (53\%) y 25 (41\%) del género masculino para cada grupo, respectivamente.

El estrato socioeconómico reveló un rango de 1 a 3 para el grupo 2 y de 2 a 6 para el grupo 1.

La frecuencia por tipo de hendidura en el grupo 2 se presenta en la Figura 1, con un predominio de la hendidura tipo 2 (36\%), seguida por la hendidura tipo 1 (24.6\%) y la tipo $3(23 \%)$.

La frecuencia de madres por grupo de estudio fue la siguiente, 13 y 58 en el grupo 1 y 2 y la frecuencia de padres, 9 y 25 , respectivamente. El promedio de edad de las madres en el momento del nacimiento de sus hijos fue de 28 y 27 para cada grupo de estudio.

\section{Análisis del acuerdo}

Los hallazgos del análisis de reproducibilidad aplicando la metodología de Bland y Altman se muestran en la Tabla 1, en la que se evidencia para la mayoría de las medidas promedios de las diferencias cercanos a cero a excepción del Go-Me. Esta diferencia cercana a 3 fue constante tanto para el grupo de padres como de madres. Un ejemplo del análisis de acuerdo se presenta en la Figura 2. 


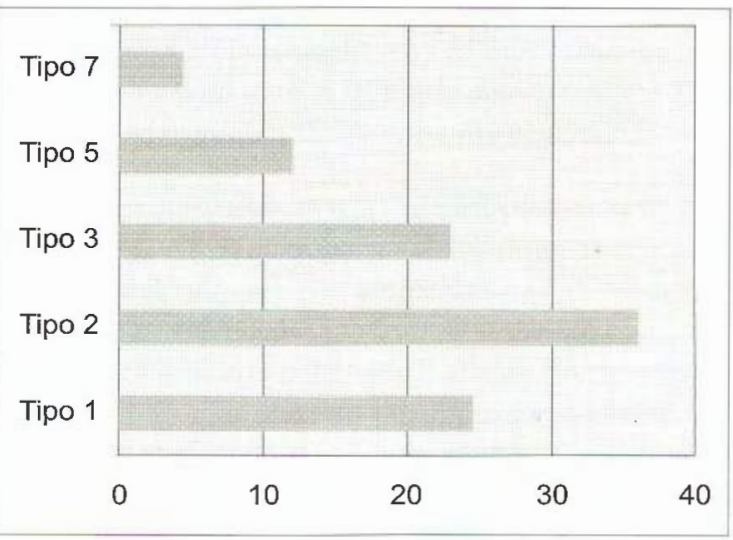

Figura 1. Descripción de la frecuencia por tipo de hendiduras.

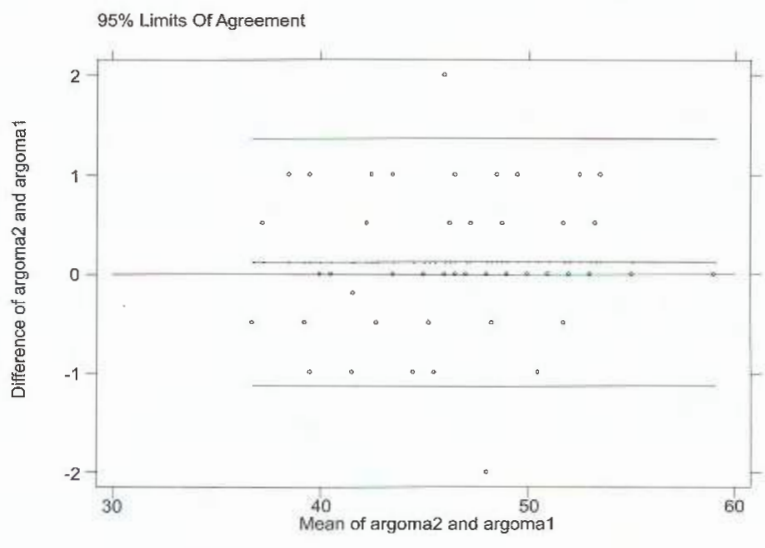

Figura 2. Análisis de reproducibilidad intra evaluador para Ar-Go en las madres.

Tabla 1. Descripción de la reproducibilidad entre-valuador en las medidas registradas. (Análisis del acuerdo de Bland y Altman)

\begin{tabular}{|c|c|c|c|c|}
\hline \multirow[b]{2}{*}{ Medida } & \multicolumn{2}{|c|}{ Mamá } & \multicolumn{2}{|c|}{ Papá } \\
\hline & $\begin{array}{c}\text { Promedio de diferencias } \\
\pm \text { DE } \\
\text { Eval } 1 \text { - Eval } 2\end{array}$ & IC $95 \%$ & $\begin{array}{c}\text { Promedio de } \\
\text { diferencias } \pm \text { DE } \\
\text { Eval } 1 \text { - Eval } 2\end{array}$ & IC $95 \%$ \\
\hline $\mathrm{CO}-\mathrm{A}$ & $0.324 \pm 0.537$ & $-0.728,1.376$ & $0.332 \pm 0.611$ & $-0.866,1.531$ \\
\hline ENA-ENP & $0.190 \pm 0.473$ & $-0.738,1.118$ & $0.309 \pm 0.508$ & $-0.686,1.304$ \\
\hline $\mathrm{Co}-\mathrm{Gn}$ & $0.455 \pm 0.621$ & $-0.762,1.672$ & $0.559 \pm 0.587$ & $-0.592,1.710$ \\
\hline Go-Me & $3.549 \pm 3.390$ & $-3.096,10.19$ & $3.044 \pm 3.110$ & $-3.051,9.140$ \\
\hline Ar-Go & $0.117 \pm 0.636$ & $-1.130,1.364$ & $0.153 \pm 0.729$ & $-1.276,1.582$ \\
\hline S-N & $0.256 \pm 0.452$ & $-0.629,1.141$ & $0.221 \pm 0.430$ & $-0.622,1.063$ \\
\hline $\mathrm{Ar}-\mathrm{Go}-\mathrm{Gn}$ & $-0.283 \pm 0.744$ & $-1.741,1.175$ & $-0.418 \pm 0.658$ & $-1.708,0.873$ \\
\hline SNA & $0.021 \pm 0.517$ & $-0.991,1.034$ & $-0.074 \pm 0.524$ & $-1.101,0.954$ \\
\hline SNB & $0.010 \pm 0.475$ & $-0.922,0.942$ & $-0-118 \pm 0.427$ & $-0.955,0.720$ \\
\hline ANB & $0.035 \pm 0.455$ & $-0.856,0.926$ & $0.059 \pm 0.457$ & $-0.836,0.954$ \\
\hline N-ENA & $0.089 \pm 0.739$ & $-1.360,1.537$ & $0.062 \pm 0.419$ & $-0.759,0.882$ \\
\hline ENA-Me & $-0.200 \pm 0.811$ & $-1.790,1.390$ & $-0.162 \pm 0.699$ & $-1.532,1.209$ \\
\hline
\end{tabular}

\section{Error Estándar de la medición}

Al analizar los datos del error estándar de la medición obtenidos para cada una de las medidas cefalométricas, se encontró un rango mínimo de 0.32 y máximo de 1.26 , lo cual no se considera clínicamente significativo (Tabla 2).

Comparación de las medidas cefalométricas por grupo de estudio.

\section{Análisis de las madres}

El análisis de los promedios de las medidas de las mamás por grupo de estudio se muestra en la Tabla 3. Se encontraron diferencias estadísticamente significativas para Co-A, ENA, Go-Me y $\mathrm{S}-\mathrm{N}$ y SNB (Figura 2 y 3). Es importante mencionar que las medidas se distribuyeron normalmente.

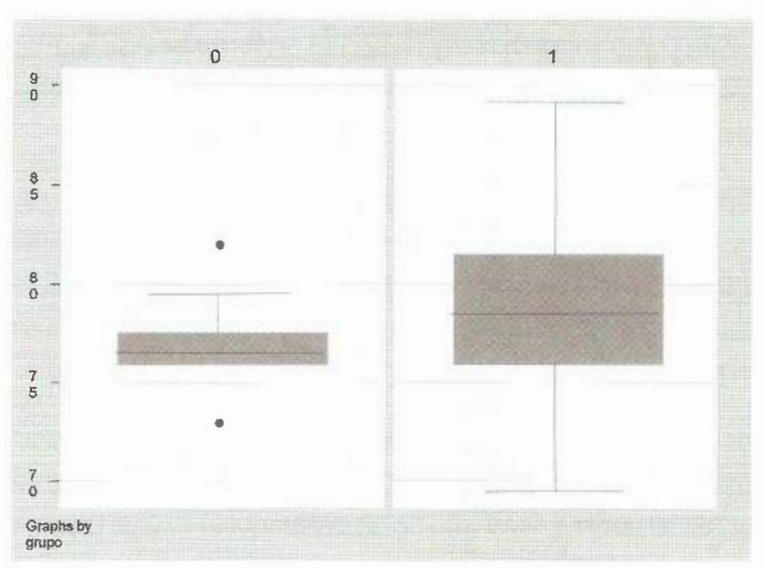

Figura 3. Comparación de la medida SNB entre las mamás de los dos grupos. 
Tabla 2. Descripción de los promedios y errores estándar de la medición para cada una de las medidas registradas.

\begin{tabular}{cccc} 
Medida & $\begin{array}{c}\text { Promedio de los } \\
\text { dos evaluadores }\end{array}$ & $\begin{array}{c}\text { Error Estándar } \\
\text { de la medición }\end{array}$ & $\begin{array}{c}\text { IC 95\% del } \\
\text { promedio }\end{array}$ \\
\hline Co-A mamá & 90.0 & 0.50 & $89.0-91.0$ \\
Co-A papá & 96.0 & 0.71 & $94.6-97.5$ \\
ENA-ENP mamá & 53.0 & 0.44 & $52.1-53.9$ \\
ENA-ENP papá & 56.8 & 0.52 & $55.8-57.9$ \\
Co-Gn mamá & 118.9 & 0.94 & $117.0-120.8$ \\
Co-Gn papá & 126.7 & 1.02 & $124.6-128.8$ \\
Go-Me mamá & 69.9 & 0.50 & $68.9-70.9$ \\
Go-Me papá & 74.8 & 0.93 & $72.9-76.7$ \\
Ar-Go mamá & 47.0 & 0.55 & $45.9-48.1$ \\
Ar-Go papá & 53.0 & 0.72 & $51.6-54.5$ \\
S-N mamá & 69.6 & 0.35 & $68.9-70.3$ \\
S-N papá & 73.3 & 0.59 & $72.1-74.5$ \\
Ar-Go-Gn mamá & 128.2 & 0.75 & $126.7-129.7$ \\
Ar-Go-Gn papá & 126.2 & 1.09 & $14.0-128.4$ \\
SNA mamá & 82.4 & 0.50 & $81.4-83.4$ \\
SNA papá & 84.3 & 0.69 & $82.8-85.6$ \\
SNB mamá & 78.6 & 0.48 & $77.6-79.5$ \\
SNB papá & 81.1 & 0.77 & $79.5-82.6$ \\
ANB mamá & 3.9 & 0.32 & $3.2-4.5$ \\
ANB papá & 3.1 & 0.42 & $2.3-4.0$ \\
N-ENA mamá & 54.3 & 0.41 & $53.5-55.1$ \\
N-ENA papá & 56.9 & 0.63 & $55.6-58.2$ \\
ENA-Me mamá & 71.4 & 0.64 & $70.2-72.7$ \\
ENA-Me papá & 74.5 & 1.26 & $71.9-77.1$ \\
\hline mann & & &
\end{tabular}

Tabla 3. Evaluación de las diferencias en las medidas registradas, según los dos grupos de estudio. Análisis de madres.

\begin{tabular}{ccccc}
\hline \multirow{2}{*}{ Medida } & $\begin{array}{c}\text { Grupo 1 } \\
\text { N:13 }\end{array}$ & $\begin{array}{c}\text { Grupo 2 } \\
\text { N.58 }\end{array}$ & $\begin{array}{c}\text { Diferencia } \\
\text { (IC95\%) }\end{array}$ & P \\
\cline { 2 - 3 } & Promedio (DE) & Promedio (DE) & & \\
\hline & & & & \\
Co-A & $92.8(4.9)$ & $89.4(3.9)$ & $3.4(0.9,5.9)$ & 0.008 \\
ENA-ANP & $55.5(2.8)$ & $52.5(3.7)$ & $3.1(0.9,5.2)$ & 0.006 \\
Co-Gn & $119.9(7.3)$ & $118.7(8.13)$ & $1.2(-3.7,6.1)$ & 0.62 \\
Go-Me & $72.1(3.9)$ & $69.4(4.1)$ & $2.6(0.1,5.2)$ & 0.04 \\
Ar-G0 & $48.2(4.7)$ & $46.7(4.6)$ & $1.5(-1.3,4.3)$ & 0.29 \\
S-N & $72.4(3.5)$ & $69.0(2.4)$ & $3.4(1.8,5.0)$ & 0.0001 \\
Ar-Go-Gn & $127.6(4.3)$ & $128.3(6.7)$ & $-0.70(-4.6,3.2)$ & 0.72 \\
SNA & $81.2(3.3)$ & $82.7(4.4)$ & $-1.4(-4.0,1.2)$ & 0.27 \\
SNB & $76.6(2.6)$ & $79(4.2)$ & $-2.4(-4.8,0.01)$ & 0.05 \\
ANB & $4.8(2.2)$ & $3.7(2.8)$ & $1.1(-0.6,2.7)$ & 0.20 \\
N-ENA & $55.9(2.5)$ & $53.9(3.5)$ & $1.9(-0.1,4.0)$ & 0.07 \\
ENA-Me & $72.9(8.2)$ & $71.1(4.6)$ & $1.8(-1.5,5.1)$ & 0.28 \\
\hline
\end{tabular}

Tabla 4. Evaluación de las diferencias en las medidas registradas, según los dos grupos de estudio. Análisis de padres.

\begin{tabular}{|c|c|c|c|c|}
\hline \multirow[t]{2}{*}{ Medida } & $\begin{array}{c}\text { Grupo } 1 \\
N: 9\end{array}$ & $\begin{array}{c}\text { Grupo } 2 \\
\mathrm{~N}: 25\end{array}$ & \multirow{2}{*}{$\begin{array}{l}\text { Diferencia } \\
\text { (IC95\%] }\end{array}$} & \multirow[t]{2}{*}{$\mathbf{P}$} \\
\hline & $\begin{array}{l}\text { Promedio } \\
\text { (DE) }\end{array}$ & Promedio (DE) & & \\
\hline $\mathrm{Co}-\mathrm{A}$ & $96.4(4.3)$ & $95.9(4.2)$ & $0.48(-2.8,3.8)$ & 0.77 \\
\hline ENA-ENP & $56.8(2.9)$ & $56.8(3.1)$ & $-0.04(-2.5,2.4)$ & 0.97 \\
\hline Co-Gn & $125.8(7.8)$ & $127.0(5.3)$ & $-1.26(-6.0,3.5)$ & 0.59 \\
\hline Go-Me & $74.4(6.7)$ & $75.0(5.1)$ & $-0.57(-4.9,3.8)$ & 0.79 \\
\hline Ar-Go & $52.8(3.2)$ & $53.1(4.5)$ & $-0.24(-3.6,3.1)$ & 0.88 \\
\hline S-N & $75.1(3.1)$ & $72.7(3.4)$ & $2.4(-0.2,5.0)$ & 0.07 \\
\hline $\mathrm{Ar}-\mathrm{G} 0-\mathrm{Gn}$ & $128.2(4.8)$ & $125.5(6.7)$ & $2.71(-2.3,7.7)$ & 0.28 \\
\hline SNA & $83.4(2.3)$ & $84.6(4.5)$ & $-1.2(-4.4,2.0)$ & 0.46 \\
\hline SNB & $80.3(2.3)$ & $81.4(5.1)$ & $-1.1(-4.7,2.5)$ & 0.52 \\
\hline ANB & $3.0(2.1)$ & $3.1(2.5)$ & $-0.2(-2.2,1.9)$ & 0.87 \\
\hline N-ENA & $56.7(3.4)$ & $56.9(3.7)$ & $-0.3(-3.3,2.6)$ & 0.83 \\
\hline ENA-Me & $76.4(5.5)$ & $73.8(7.9)$ & $2.6(-3.2,8.5)$ & 0.37 \\
\hline
\end{tabular}

\section{Análisis de padres}

Al comparar las medidas cefalométricas por grupo de estudio en la muestra de padres no se detectaron diferencias estadisticamente significativas (Tabla 4).

\section{Análisis de las medidas por tipo de hendidura}

Al comparar los promedios de las medidas cefalométricas por tipo de hendidura para el grupo de madres se muestra en la Tabla 5. Solamente se detectaron diferencias significativas para el SNA y el SNB (Figura 3). El análisis para el grupo de padres no registró diferencias significativas (Tabla 6).

\section{DISCUSIÓN}

La reproducibilidad entre evaluadores de las medidas registradas es alta, con un índice de confiabilidad del 95\% según la prueba de Bland y Altman lo cual le confiere validez al estudio. La mayor diferencia se encontró en la medida Go-Me, posiblemente por la dificultad en la localización en el punto Me.

La opinión contemporánea acerca de la etiología de las hendiduras de labio y/o paladar, es que son de origen poligénico y multifactorial, siendo influenciadas en gran parte por un componente ambiental. La relativa contribución de cada uno de estos factores para cada caso en particular es desconocida.

Los estudios sobre morfología craneofacial de padres de niños con hendiduras han reportado diferencias que los distinguen de la población en general. ${ }^{10-14}$ Sin embargo, hay diferencias en los resultados de los diversos estudios, que se pueden explicar debido a diferencias metodológicas, de tamaño en la muestra, pero también en parte debido a la heterogeneidad en la etiología de las hendiduras orofaciales. También se podría relacionar que las diferencias en algunas variables craneofaciales están bajo mayor control genético que otras.

Ward y colaboradores sugieren que la falta de acuerdo entre los estudios se podría deber al modelo de umbral multifactorial. ${ }^{17}$ Este modelo asume que ambos padres contribuyen en iguales proporciones en el niño afectado y en los resultados no aparece que un padre contribuya más que otro.

En el presente estudio, se discriminaron en grupos padres y madres, pero por razones socioculturales, la muestra de las madres fue mayor que la de los padres en los dos grupos de estudio, esto podría tener alguna influencia en los resultados ya que las diferencias encontradas fueron estadísticamente significativas $(\mathrm{P}<0.05)$ en el grupo de madres pero no en el de padres. A diferencia de Prochazkova y Tolarova que no reportaron diferencias de género. ${ }^{18}$ Por su parte, Blanco comparó sujetos afectados con labio y/o paladar hendido, encontrando ciertos parámetros de diferencia solo en mujeres. ${ }^{19}$ Muchos estudios no separan madres de padres en el análisis de sus resultados y no hay registro de la relación madre:padres en la susceptibilidad de las hendiduras. 
Tabla 5. Análisis de varianza de las medidas registradas por tipo de hendidura Análisis de madres

\begin{tabular}{|c|c|c|c|c|c|c|}
\hline \multirow{3}{*}{ Medida } & \multicolumn{5}{|c|}{ Tipo de hendidura } & \multirow[t]{3}{*}{$\mathbf{p}$} \\
\hline & 1 & 2 & 3 & 5 & 7 & \\
\hline & $\mathrm{N}: 14$ & $\mathrm{~N}: 18$ & N:13 & $\mathrm{N}: 2$ & N:6 & \\
\hline $\mathrm{Co}-\mathrm{A}$ & 90.5 & 90.1 & 88.4 & 85.9 & 89.8 & 0.43 \\
\hline ENA-ENP & 52.9 & 53.0 & 51.2 & 49.3 & 52.3 & 0.50 \\
\hline Co-Gn & 119.6 & 121.3 & 115.6 & 116.8 & 118.9 & 0.47 \\
\hline Go-Me & 69.4 & 69.5 & 69.8 & 68.2 & 70.2 & 0.99 \\
\hline Ar-Go & 47.6 & 47.7 & 44.6 & 47.4 & 46.4 & 0.40 \\
\hline S-N & 68.1 & 69.9 & 68.5 & 68.0 & 69.0 & 0.28 \\
\hline $\mathrm{Ar}-\mathrm{Go}-\mathrm{Gn}$ & 127.6 & 129.4 & 128.4 & 134.5 & 127.0 & 0.68 \\
\hline SNA & 83.9 & 83.3 & 79.4 & 80.0 & 96.1 & 0.004 \\
\hline SNB & 79.4 & 79.9 & 75.9 & 78 & 83.4 & 0.001 \\
\hline ANB & 4.5 & 3.9 & 3.5 & 2.0 & 2.7 & 0.63 \\
\hline N-ENA & 53.2 & 54.4 & 55.1 & 55.4 & 50.5 & 0.08 \\
\hline ENA-Me & 72.3 & 71.2 & 70.8 & 74.8 & 67.6 & 0.23 \\
\hline
\end{tabular}

Tabla 6. Análisis de varianza de las medidas registradas por tipo de hendidura Análisis de padres.

\begin{tabular}{|c|c|c|c|c|c|}
\hline \multirow{3}{*}{ Medida } & \multicolumn{4}{|c|}{ Tipo de hendidura } & \multirow{3}{*}{$\mathbf{p}$} \\
\hline & 1 & 2 & 3 & 7 & \\
\hline & $\mathrm{N}: 6$ & $\mathrm{~N}: 8$ & N:4 & N:5 & \\
\hline $\mathrm{CO}-\mathrm{A}$ & 94.6 & 96.7 & 93.3 & 96.3 & 0.53 \\
\hline ENA-ENP & 55.2 & 57.8 & 54.0 & 58.3 & 0.07 \\
\hline $\mathrm{Co}-\mathrm{Gn}$ & 125.4 & 128.1 & 121.4 & 128.2 & 0.08 \\
\hline Go-Me & 73.4 & 76.8 & 71.4 & 74.3 & 0.28 \\
\hline Ar-Go & 50.2 & 54.1 & 52.1 & 53.0 & 0.40 \\
\hline S-N & 72.9 & 73.1 & 70.9 & 71.6 & 0.66 \\
\hline $\mathrm{Ar}-\mathrm{G} 0-\mathrm{Gn}$ & 124.0 & 123.9 & 127.0 & 129.2 & 0.52 \\
\hline SNA & 80.7 & 86.1 & 83.3 & 86.5 & 0.08 \\
\hline SNB & 77.6 & 82.2 & 81.0 & 82.9 & 0.30 \\
\hline ANB & 3.0 & 3.9 & 2.3 & 3.6 & 0.76 \\
\hline N-ENA & 58.1 & 56.8 & 56.0 & 56.9 & 0.89 \\
\hline ENA-Me & 77.4 & 73.9 & 65.1 & 76.3 & 0.10 \\
\hline
\end{tabular}

En el presente estudio, se encontraron diferencias estadísticamente significativas en la medida de la longitud del maxilar superior ( $\mathrm{Co}-\mathrm{A})$ en las madres, siendo más corta en el grupo 1 (grupo de madres con hijos afectados) que en el grupo 2. En los padres no se encontró diferencia estadísticamente significativa, posiblemente, por el tamaño de la muestra. Nakasima e Ichinose encontraron dimensiones más largas de la cara superior pero medida en una radiografía frontal. ${ }^{13}$

La longitud de maxilar superior medida desde espina nasal posterior a espina nasal anterior (ENA-ENP) se encontró disminuida de manera estadísticamente significativa $(\mathrm{p}<0.05)$ en las madres de los niños afectados respecto a las madres con hijos sin hendiduras, coincidiendo con lo reportado por Coccaro y colaboradores, y Nakashima e Ichonese quienes igualmente encontra- ron disminuido su tamaño. ${ }^{9,20}$ De otra parte, Raghavan y colaboradores encontraron que los padres de niños con hendiduras orofaciales tienen una longitud maxilar (ENA-ENP) significativamente más larga. ${ }^{21}$

En cuanto a la longitud mandibular, Mossey y colaboradores encontraron una mandíbula más grande en las madres de los niños con LPH. ${ }^{22}$ En el presente estudio se encontró la medida del cuerpo mandibular ( $\mathrm{Go}-\mathrm{Me}$ ) disminuida y fue estadísticamente significativa, teniendo en cuenta que esta medida fue la que tuvo mayor rango de error interevaluador. Al medir $\mathrm{Co}$-Gn en las madres, también se encontró reducida pero no de manera estadísticamente significativa. Prochazkova y Tolarova también encontraron disminuida esta medida en el grupo de estudio. ${ }^{11}$ 
En este estudio, la longitud de la base del cráneo (S-N) de las madres de hijos con hendiduras se encontró disminuida de manera estadísticamente significativa $(p<0.01)$ a diferencia de lo reportado por Mossey y colaboradores, y Raghavan y colabores que no encontraron ninguna diferencia respecto a esta medida entre los dos grupos. ${ }^{21,22}$

En el estudio realizado por Sato, el ángulo SNA fue levemente mayor en todos los padres. ${ }^{23}$ Coincide con los resultados obtenidos en este estudio en donde el ángulo SNA fue levemente mayor tanto en padres como en madres aunque de manera no estadísticamente significativa.

El ángulo SNB, en este estudio, se encontró disminuido de manera estadísticamente significativa $(p<0.05)$ en las madres de niños con hendiduras mientras que Raghavan y colaboradores no encontraron diferencia significativa entre los dos grupos para esta medida. ${ }^{21}$

De las dos anteriores medidas se desprende el ángulo ANB, que estaba disminuido en las madres con hijos afectados, no así en los padres, lo que podría sugerir, tendencia a un perfil prognático en las madres de hijos con LPH.

\section{CONCLUSIONES}

Los resultados obtenidos soportan las siguientes conclusiones:

1. Los datos encontrados arrojan muy buena reproducibilidad interevaluador en la medición lo cual le da gran confiabilidad y validez a este estudio. El error estándar de la medición fue muy bajo y no se considera clínicamente significativo.

2. Existen diferencias significativas en la morfología craneofacial de madres de niños con hendiduras orofaciales al ser comparadas con el grupo de madres de niños sin hendiduras, no sucede lo mismo con el grupo de padres, lo que podría sugerir una mayor influencia materna en la genética de la morfología craneofacial y en la predisposición a tener hijos con labio y/o paladar hendido.

3. Las medidas diferentes estadísticamente significativas fueron: Co - A, ENA - ENP, Go- Me, S-N, Angulo SNB $(\mathrm{P}<0.05)$. Lo cual coincide con algunos estudios de la literatura.

4. No hubo diferencias significativas en las medidas cefalométricas de los padres.

5. Aunque existe evidencia de que la morfología craneofacial de los padres de niños con LPH difiere de la morfología craneofacial de padres de niños sanos, la información disponible es insuficiente para localizar exactamente esas diferencias.

6. Al comparar las madres de niños enfermos, según el tipo de hendidura se encontraron diferencias significativas en SNA y SNB, esta diferencia la aporta la hendidura 3 ( labio y paladar hendido bilateral).
7. Los mayores problemas respecto a la variabilidad de registros encontrados se asocian a las diferencias metodológicas entre las investigaciones

\section{BIBLIOGRAFÍA}

1. Murria JC. Gene/enviroment of cleft lip and/or palate. Clin Genet 2002; 61: $248-256$.

2. Muñoz J, Bustos I, Quintero C, Giraldo A. Factores de riesgo para algunas anomalías congénitas en la población colombiana. Revista de Salud Pública 2003. URL disponible en: http:// www.medicina.unal.edu.co

3. Ward R, Bixler D, Jamison P. Cephalometric evidence for a dominantly inherited predisposition to cleft lip - cleft palate in a single large kindred. Am J Med Genet 1994; 50: 57 - 63.

4. Scapoli L, Martinelli M, Pezzetti F, Carinci F, Bodo M, Tognon M, Carinci P. Linkage disequilibrium between GABREB3 gene and nonsyndromic familial cleft lip with or without cleft palate. Hum Genet 2002; 110: 15 - 20.

5. Thorogood P, Ferreti P. Heads and tales: recente advances in craniofacial development. Br Dent J 1992; 173: $301-306$.

6. Wyszynski DF, Beaty TH. Review of the role of potential teratogens in the origin of human nonsyndromic oral cleft. Teratology 1996; 53: $309-317$.

7. Bianchi F, Calzolari E, Ciulli L, Cordier S, Gualandi F, Pierini A, Mossey P. Environment and genetics in the etiology of cleft lip and cleft palate with reference to the role of folic acid. Epidemiol Prev $2000 ; 24: 21-27$.

8. Mossey PA, McColl JH, Stirrups DR. Differentiation between cleft lip with or without cleft palate and isolated cleft palate using parental cephalometric parameters. Cleft Palate Craniofac J 1997; 34: $27-35$.

9. Coccaro PJ, D'Amico R, Chavoor A. Craniofacial morphology of parents with and without lip and palate children. Cleft Palate J 1972; 9: $28-42$.

10. Nakasima A, Ichinose M Characteristics of craniofacial structures on parents of children with cleft lip and/or palate. Am J Orthod 1983; 84: $140-146$.

11. Prochazkova J, Vinsova J. Craniofacial morphology as a marker of predisposition to isolated cleft palate. J Craniofac Genet Dev Biol 1995; 15: $162-168$.

12. Peters $\mathrm{H}$, Neubuser A, Kratochwil K, Balling R. Pax 9 deficient mice lack pharyngeal pouch derivatives and teeth and exhibit craniofacial and limb abnormalities. Gene Dev 1998; 12: 2735 - 2747.

13. McIntire GT, Mossey PA. The craniofacial morphology of the parents of children with orofacial clefting: a systematic review of cephalometric studies. J Orthod 2002; 29: $23-29$.

14. Mcintire GT, Mossey PA. Asymmetry of the parental craniofacial skeleton in orofacial clefting. J Orthod 2002; 29: 299- 305.

15. Bland M, Altman D. Statistical methods for assessing agreement between two methods of clinical measurements. Lancet 1986; 8: 307 - 310. 
16. Pagano M, Gauvreau K. Principles of biostatistics. Duxbury Press, Belmont Ca, 1993.

17. Ward RE, Buxer D, Raywood ER. A study of cephalometric features in cleft lip-cleft palate families. I: Phenotypic heterogeneity and genetic predisposition in parents of sporadic cases. Cleft Palate J 1989; $26: 318-325$.

18. Prochazkva J, Tolarova M. Craniofacial morphological features in parents of children with isolated cleft palate. Acta Chir Plast 1986; 28: $194-204$.

19. Blanco R, Cifuentes L, Maldonado MJ, Rameau MX, Muñoz MA. Cleft lip and cleft palate, cephalometrics features of affected individuals, their relatives and a control population. Rev Med Chile 1992; 120: $13-19$.

20. Nakasima $A$, Ichinose $M$. Size in cranium in parents and their children with cleft lip. Cleft Palate J 1984; 21: 193-203.

21. Raghavan R, Sidhu SS, Kharbanda OP. Craniofacial pattern of parents of children having cleft lip and/or cleft palate anomaly. Angle Orthod 1994; 64: 137 - 144.

22. Mossey PA, McColl J, O'Hara M. Cephalometric features in the parents of children with orofacial clefting. Br J Oral Maxillofacial Surg 1998; 36: $202-212$.

23. Sato T. Craniofacial morphology of parents with cleft lip and palate children. Shikwa Gakuho 1989; 89: 1479 - 1506 